\title{
Study of Soluble HLA-G in Congenital Human Cytomegalovirus Infection
}

\author{
Roberta Rizzo, ${ }^{1}$ Liliana Gabrielli, ${ }^{2}$ Daria Bortolotti, ${ }^{1}$ Valentina Gentili, ${ }^{1}$ \\ Giulia Piccirilli, ${ }^{2}$ Angela Chiereghin, ${ }^{2}$ Claudia Pavia, ${ }^{2}$ Silvia Bolzani, ${ }^{1}$ Brunella Guerra, ${ }^{3}$ \\ Giuliana Simonazzi, ${ }^{3}$ Francesca Cervi, ${ }^{3}$ Maria Grazia Capretti, ${ }^{4}$ Enrico Fainardi, ${ }^{5}$ \\ Dario Di Luca, ${ }^{1}$ Maria Paola Landini, ${ }^{6}$ and Tiziana Lazzarotto ${ }^{6}$ \\ ${ }^{1}$ Department of Medical Sciences, Section of Microbiology and Medical Genetics, University of Ferrara, Ferrara, Italy \\ ${ }^{2}$ Operative Unit of Clinical Microbiology, St. Orsola-Malpighi University Hospital, Bologna, Italy \\ ${ }^{3}$ Operative Unit of Obstetrics and Prenatal Medicine, Department of Medical and Surgical Sciences, \\ St. Orsola-Malpighi University Hospital, University of Bologna, Bologna, Italy \\ ${ }^{4}$ Operative Unit of Neonatology, St. Orsola-Malpighi University Hospital, Bologna, Italy \\ ${ }^{5}$ Operative Unit of Neuroradiology, Careggi University Hospital, Firenze, Italy \\ ${ }^{6}$ Operative Unit of Clinical Microbiology, Department of Specialised, Experimental, and Diagnostic Medicine, \\ St. Orsola-Malpighi University Hospital, University of Bologna, Bologna, Italy
}

Correspondence should be addressed to Roberta Rizzo; roberta.rizzo@unife.it

Received 19 April 2016; Revised 9 August 2016; Accepted 16 August 2016

Academic Editor: Nejat K. Egilmez

Copyright ( 2016 Roberta Rizzo et al. This is an open access article distributed under the Creative Commons Attribution License, which permits unrestricted use, distribution, and reproduction in any medium, provided the original work is properly cited.

\begin{abstract}
Human leukocyte antigen-G (HLA-G) is a nonclassical HLA class I antigen that is expressed during pregnancy contributing to maternal-fetal tolerance. HLA-G can be expressed as membrane-bound and soluble forms. HLA-G expression increases strongly during viral infections such as congenital human cytomegalovirus (HCMV) infections, with functional consequences in immunoregulation. In this work we investigated the expression of soluble (s)HLA-G and beta-2 microglobulin (component of HLA) molecules in correlation with the risk of transmission and severity of congenital HCMV infection. We analyzed 182 blood samples from 130 pregnant women and 52 nonpregnant women and 56 amniotic fluid samples from women experiencing primary HCMV infection. The median levels of sHLA-G in maternal serum of women with primary HCMV infection were higher in comparison with nonprimary and uninfected pregnant women $(p<0.001)$. AF from HCMV symptomatic fetuses presented higher sHLA-G levels in comparison with infected asymptomatic fetuses $(p<0.001)$, presence of HLA-G free-heavy chain, and a concentration gradient from amniotic fluid to maternal blood. No significant statistical difference of beta- 2 microglobulin median levels was observed between all different groups. Our results suggest the determination of sHLA-G molecules in both maternal blood and amniotic fluid as a promising biomarker of diagnosis of maternal HCMV primary infection and fetal HCMV disease.
\end{abstract}

\section{Introduction}

Human cytomegalovirus (HCMV) is the most common cause of intrauterine infection, occurring in $0.3 \%$ to $2.3 \%$ of births [1]. HCMV intrauterine transmission is more common after primary infection (30-40\% of probability) than after nonprimary infection (1\%) $[2,3]$. Nevertheless, it was estimated that, for all population seroprevalences, nonprimary maternal infections are responsible for the majority of congenital CMV infections [4].

Ten to fifteen percent of congenitally infected infants of primarily infected women will have symptoms at birth and around $10 \%$ of them will not survive. Moreover, $70-80 \%$ of surviving babies will suffer delayed sequelae such as sensorineural hearing loss, delay of psychomotor development, and visual impairment [5]. Most congenital infected infants 
(85-90\%) have no symptoms at birth, but $8 \%$ to $15 \%$ of them will develop delayed injury $[3,5]$.

The fetal compartment can be studied by invasive (amniocentesis) and noninvasive (ultrasound examination) techniques [6]. Ultrasonographic findings are helpful but not diagnostic findings since HCMV has features in common with other intrauterine infections and its sensitivity is poor [7]. HCMV detection in amniotic fluid with virus isolation and/or Real-Time PCR is useful for prenatal diagnosis of fetal infection, due to its high sensitivity and specificity [8-10]. There is still a need for reliable prognostic factors for the outcome of HCMV fetal infection.

HCMV can modulate the expression and/or function of human leukocyte antigens (HLA), by encoding proteins to detain and destroy the expression of HLA molecules on the surface of infected cells, or selectively upregulate specific HLA class I molecules binding to immune cell inhibitory receptors [11]. In this scenario, there is an interesting nonclassical HLA class I antigen, HLA-G, characterized by low allelic polymorphism, restricted tissue distribution, and alternative mRNA splicing which creates different isoforms, 4 membrane-bound (HLA-G1-G4) and 3 soluble (HLA-G5G7) [12]. In addition, the HLA-G1 isoform can produce a soluble form called sHLA-G1, derived from membrane proteolytic shedding [13]. HLA-G is expressed at the maternal-fetal interface, on surface of trophoblasts [12], and the concentrations of soluble (s)HLA-G increase in the plasma samples of pregnant women during the first trimester of pregnancy [14]. HCMV infection modifies HLA-G expression in tissues and immune cells, with a downmodulation in infected cytotrophoblasts [15] and upregulation in infected peripheral blood cells [16]. Specific HCMV proteins modify HLA-G expression interacting with the HLA-G promoter, and affecting mRNA stability, protein translation, and the secretory pathway [1719]. The increase in HLA-G expression is suggested as a mechanism for virus immune escape, due to the immune-inhibitory functions of HLA-G. Finally, it has been observed that another important component of HLA class I molecules, beta-2 microglobulin (b2M), has a diagnostic efficacy for differentiating symptomatic from asymptomatic HCMV congenital infection [20].

In order to explore the possible role of HLA-G molecules in congenital HCMV infection, we analyzed maternal and fetal sHLA-G and b2M expression in correlation with the risk of transmission and severity of HCMV infection.

\section{Materials and Methods}

2.1. Subjects. The study analyzed the serum samples of a cohort of 130 pregnant women who were referred to the Maternal-Fetal Medicine Unit, St. Orsola-Malpighi University Hospital, Bologna, between 2006 and 2011 for suspected primary maternal HCMV infection. Maternal primary HCMV infection was assessed at the Virology Unit of the same University Hospital. Written informed consent for the studies was obtained from all patients according to the protocol approved by the Scientific Ethical Committee of the Ferrara and Bologna Universities.
The women, aged between 18 and 40 years, were in the first or second trimester of pregnancy. They presented no previous autoimmune and inflammatory diseases and they were not on any anti-inflammatory or immune-modulatory drugs or hyperimmune globulin.

Primary infection was diagnosed based on clinical and laboratory history and HCMV IgM-positive and low/moderate HCMV IgG avidity results as well as positive DNAemia and/or seroconversion for HCMV. Nonprimary maternal HCMV infection was diagnosed, within the first 16 weeks of gestation, according to blot-confirmed IgM-positivity with high avidity anti-HCMV IgG and presence of DNA-HCMV in blood and/or urine and/or saliva.

HCMV-seronegative women (for both IgG and IgM) were defined as uninfected.

Fifty-two nonpregnant women with HCMV past infection (IgG positive and IgM negative) were recruited as healthy controls.

Moreover, 56 amniotic fluid samples were collected during amniocentesis (20-21 weeks of gestation) from those pregnant women with primary HCMV infection arising before the 14th week of gestation; HCMV detection on amniotic fluid samples was performed with virus isolation and realtime PCR.

\subsection{Diagnosis of Congenital HCMV Infection in Fetuses and} Infants. Infection status of the aborted fetuses was classified on the basis of histological and immunohistochemical tissue examination, whereas the infection status of infants was classified on the basis of viral isolation and real-time PCR from urine within the first 2 weeks of life.

Fetal symptomatic infection was defined as the presence of ultrasound abnormalities and histological and immunohistochemical findings in fetal organs with particular attention to the brain [21]. CMV disease in infected newborns was investigated through clinical, instrumental, and laboratory examination in the neonatal and subsequently monitored up to 6 years of age [22].

2.3. Anti-HCMV IgM and IgG Detection and IgG Avidity. Maternal serum samples were tested using the Enzygnost ${ }^{\circledR}$ HCMV IgM and Enzygnost HCMV IgG assays (Siemens Healthcare Diagnostics) and an in-house immunoblot for detection of HCMV-specific IgM [23]. HCMV IgG avidity was tested with the Radim ${ }^{\circledR}$ Cytomegalovirus IgG Avidity EIA WELL assay (Radim).

2.4. Virological Examinations. HCMV isolation from amniotic fluid was performed by shell-vial procedure as described elsewhere [24]. DNA was extracted from amniotic fluid and saliva with the NucliSens easyMAG System (bioMerieux) and from blood and urine with the QIAsymphony SP/AS System (QIAGEN).

HCMV-DNA was quantified with a real-time PCR assay (HCMV ELITe MGB kit, ELITechGroup) using the ELITe MGB technology. Amplification, detection, and analysis were performed with the ABI PRISM 7300 platform (PE Applied Biosystems). The detection limit was 11 copies/reaction and 
viral load was reported as number of copies/mL for all body fluids examined.

\subsection{Enzyme-Linked Immunosorbent Assay (ELISA) for Soluble} $H L A-G$. sHLA-G levels in serum and amniotic fluid samples were assayed in triplicate as previously reported $[25,26]$ using the monoclonal antibody (MoAb) MEM-G9 (Exbio), which recognizes the HLA-G molecules, in b2M associated form. The intra-assay coefficient of variation $(\mathrm{CV})$ was $1.4 \%$ and the interassay CV was $4.0 \%$. The limit of sensitivity was $1.0 \mathrm{ng} / \mathrm{mL}$.

2.6. ELISA for Soluble Beta-2 Microglobulin and Albumin. b2M concentration was determined in triplicate using a commercial human beta- 2 microglobulin ELISA Kit (Abcam) with a detection limit $<6 \mathrm{pg} / \mathrm{mL}$.

Albumin concentration was determined in triplicate with a 1:200 dilution using the commercial human albumin ELISA Kit (Alpha Diagnostic International) with intra-assay CV of 6.8 to $11.4 \%$ and interassay CV of 3.5 to $6.4 \%$.

2.7. Determination of sHLA-G Index. Fetal production of HLA-G was calculated with the following formula [27]:

$$
\text { sHLA-G Index }=\frac{\text { amniotic fluid }: \text { serum sHLA-G }}{\text { amniotic fluid } \text { serum albumin }}
$$

where the ratio between amniotic fluid and serum albumin concentrations represents the status of placental barrier.

2.8. Western Blot Analysis. Serum samples and amniotic fluids were biotinylated with $0.2 \mathrm{mg} / \mathrm{mL}$ EZ-Link Sulfo-NHSLC-Biotin (Pierce) in $\mathrm{pH} 8.0 \mathrm{PBS} 1 \mathrm{x}$ for $30 \mathrm{~min}$ at $4^{\circ} \mathrm{C}$ [26]. Samples were then immunoprecipitated for $2 \mathrm{hrs}$ at room temperature with anti-HLA-G MoAb (MEM-G1, specific for HLA-G free-heavy chain, or MEM-G9, specific for b2M conjugated HLA-G, Exbio), washed twice in PBS 1x, and incubated overnight with protein G-Sepharose beads (Santa Cruz) at $4^{\circ} \mathrm{C}$. The samples were washed twice and resuspended in $20 \mu \mathrm{L}$ Laemmli Buffer (BioRad). We quantified protein concentration in immunoprecipitates by the Bradford assay (BioRad Laboratories) using bovine albumin (Sigma-Aldrich) as standard. Total protein was denatured at $100^{\circ} \mathrm{C}$ for $5 \mathrm{~min}$. Proteins were loaded with native or reducing buffers in 10\% TGXPrecast gel (Biorad), with subsequent electroblotting transfer onto a PVDF membrane (Millipore) [28]. The membrane was incubated with a horseradish peroxidase- (HRP-) conjugated antimouse antibody (1:5000; Amersham Biosciences) and developed with the ECL kit (Amersham Biosciences). The images were acquired by Geliance 600 (Perkin Elmer).

2.9. Statistics. Statistical analysis was performed with Stat View software package (SAS Institute Inc). Given that the data, screened by Kolmogorov-Smirnov test, presented a normal distribution, statistical analyses were performed using Student's $t$-test. Frequencies of positive samples for a specific variable were compared by Fisher exact test. A logistic regression analysis was performed to evaluate the effect of different variables. The relationship between sHLAG presence and HCMV infection status was investigated by the Receiver Operating Characteristic (ROC) curve analysis (JROCFIT software, John Hopkins University).

\section{Results}

3.1. sHLA-G and Beta-2 Microglobulin Levels in Maternal Serum Samples. We evaluated sHLA-G and b2M levels in sera of 130 pregnant women, 30 uninfected, 56 with primary HCMV infection, and 44 with nonprimary HCMV infection, and 52 nonpregnant women with HCMV past infection.

Detectable serum levels of sHLA-G were significantly more frequent in pregnant $(130 / 130 ; 100 \%)$ than in nonpregnant women $(31 / 52 ; 59.6 \%)(p<0.0001)$. b2M molecules presented only slightly higher positive samples in pregnant women $(65 / 100 ; 50 \%)$ than in nonpregnant women $(20 / 52$; $38.5 \%)(p=0.14)$ (data not shown).

Regarding the median levels of sHLA-G, pregnant women showed higher levels of molecules in comparison with nonpregnant women ( 49 versus $21 \mathrm{ng} / \mathrm{mL}$; range: $37.4-$ $76.5 \mathrm{ng} / \mathrm{mL}$ versus $0.0-20.5 \mathrm{ng} / \mathrm{mL})$ irrespective of HCMV infection $(p<0.001)$ (Figure 1(a)). In addition, we observed no statistical differences in sHLA-G serum median levels between actively HCMV infected (56 with primary and 44 with nonprimary infection) and 30 uninfected pregnant women (47 versus $50 \mathrm{ng} / \mathrm{mL}$; range: $36.2-69.8$ versus $37.4-$ $76.5 \mathrm{ng} / \mathrm{mL} ; p=0.43$ ) (data not shown).

Interestingly, subdividing the subjects according to the maternal HCMV infection status, primary infected pregnant women presented higher levels of sHLA-G median concentrations $(62 \mathrm{ng} / \mathrm{mL} ; 46.9-69.8 \mathrm{ng} / \mathrm{mL})$ than nonprimary infected women $(44 \mathrm{ng} / \mathrm{mL} ; 36.2-57.2 \mathrm{ng} / \mathrm{mL})(p<$ 0.001 ). Moreover, primary infected pregnant women presented higher sHLA-G median concentrations than uninfected women $(50 \mathrm{ng} / \mathrm{mL} ; 37.4-76.5 \mathrm{ng} / \mathrm{mL})(p=0.006)$ (Figure 1(a)).

The median levels of b2M were only slightly higher in actively HCMV infected (primary and nonprimary) than in uninfected pregnant women (1.8 versus $1.3 \mu \mathrm{g} / \mathrm{mL} ; p=0.14$ ), in primary than in nonprimary HCMV infected (1.9 versus $1.4 \mu \mathrm{g} / \mathrm{mL} ; p=0.12$ ), and, finally, in primary HCMV infected than in uninfected women ( 1.9 versus $1.3 \mu \mathrm{g} / \mathrm{mL} ; p=0.06$ ) (Figure 1(b)).

3.2. sHLA-G and Beta-2 Microglobulin Levels in Amniotic Fluid. We evaluated sHLA-G and b2M levels in 56 amniotic fluid samples from women who were primarily HCMV infected before week 14 of gestation and accepted amniocentesis.

Out of the 56 amniotic fluids, 39 samples were HCMV negative with PCR and virus isolation and no congenitally infected newborns were found in this group.

Out of 17 amniotic fluids from mothers who transmitted the virus to their fetuses/babies, two were negative for both virus isolation and PCR. Despite these negative results, the 2 babies were congenitally infected, but asymptomatic at birth and during the follow-up period, as already described in the literature $[9,10]$. The remaining 15 amniotic samples were positive for both virological tests $\left(6 \times 10^{5}\right.$ copies $/ \mathrm{mL}$ median 


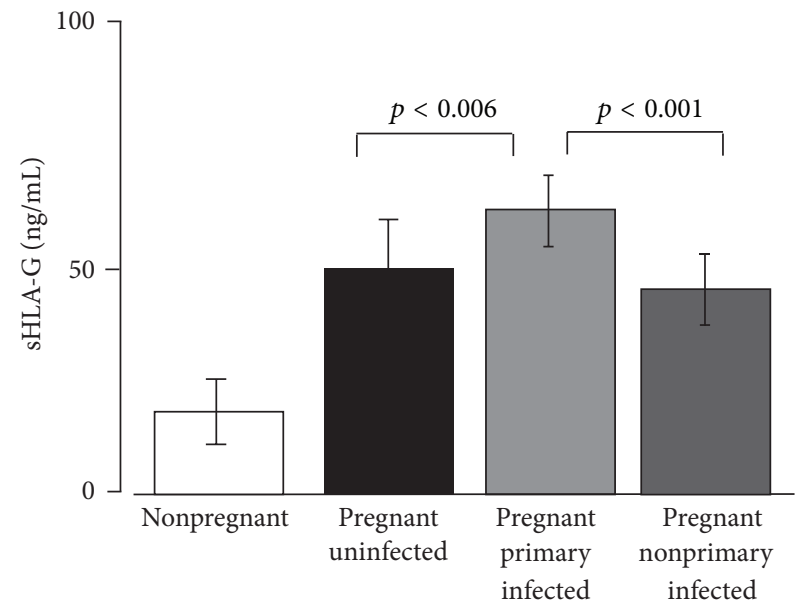

(a)

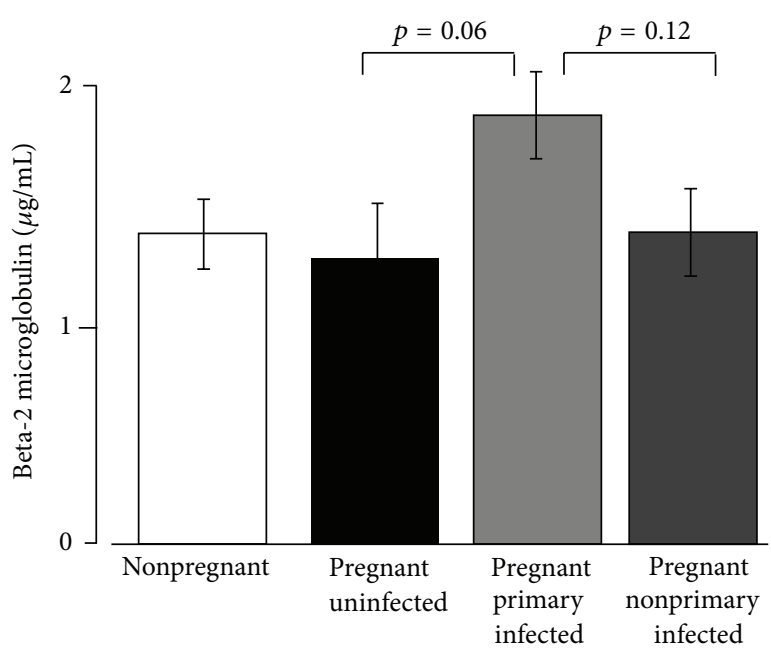

(b)

FIGURE 1: Maternal serum samples expression of (a) sHLA-G and (b) b2M molecules in nonpregnant and pregnant women. Pregnant women are classified as uninfected and primarily and nonprimarily HCMV infected. $p$ values obtained by Student's $t$-test and mean \pm standard deviation are reported.

viral load) except for one case where only HCMV-DNA was detected $\left(10^{3}\right.$ copies $\left./ \mathrm{mL}\right)$.

Overall, out of the 17 fetuses/babies infected with congenital HCMV, 5 newborns were asymptomatic at birth and during subsequent monitoring and 11 fetuses and 1 newborn were symptomatic.

The brains of all symptomatic fetuses were HCMV positive with severe histological brain damage and cerebral necrosis; 4 of the fetuses also showed pathological neurosonographic findings (periventricular hyperechogenicity and ventriculomegaly).

Moreover, the only symptomatic newborn had hepatosplenomegaly, thrombocytopenia (platelet count: $<100.000 / \mathrm{mm}^{3}$ ), and alanine aminotransferase elevation $(>80 \mathrm{U} / \mathrm{L})$ at birth and developed sequelae with sensorineural hearing loss and mild psychomotor retardation.

No statically significant difference in amniotic fluid HCMV load was observed between asymptomatic and symptomatic fetuses/newborns ( $p=0.88,95 \% \mathrm{CI}:-3583021$ to 3110188).

All the 56 amniotic fluid samples were positive for sHLA$\mathrm{G}$ and $\mathrm{b} 2 \mathrm{M}$ molecules (data not shown).

Median detectable levels of sHLA-G were significantly higher in amniotic fluids from infected symptomatic fetuses $(73 \mathrm{ng} / \mathrm{mL} ; 69-79 \mathrm{ng} / \mathrm{mL})$ than in infected asymptomatic fetuses $(32 \mathrm{ng} / \mathrm{mL} ; 28-42 \mathrm{ng} / \mathrm{mL})(p<0.001)$ and in uninfected fetuses $(31 \mathrm{ng} / \mathrm{mL} ; 29-40.2 \mathrm{ng} / \mathrm{mL})(p<0.001)$ (Figure 2(a)).

b2M presented slightly higher median levels in amniotic fluids from infected symptomatic fetuses $(4.5 \mu \mathrm{g} / \mathrm{mL})$ than in infected asymptomatic fetuses $(3.6 \mu \mathrm{g} / \mathrm{mL})(p=0.039)$ and uninfected fetuses $(3.9 \mu \mathrm{g} / \mathrm{mL})(p=0.042)$ (Figure 2(b)).

When we considered maternal serum levels according to fetus infection status, we observed that sHLA-G concentrations were slightly higher in serum from women with symptomatic fetuses $(51.2 \mathrm{ng} / \mathrm{mL}, 45-57 \mathrm{ng} / \mathrm{mL})$ and in women with infected asymptomatic fetuses ( $49 \mathrm{ng} / \mathrm{mL}, 42-55 \mathrm{ng} / \mathrm{mL}$ ) in comparison with uninfected fetuses $(46 \mathrm{ng} / \mathrm{mL}, 44-$ $52 \mathrm{ng} / \mathrm{mL})(p=0.045, p=0.042$, resp.) (Figure 2(c)).

$\mathrm{b} 2 \mathrm{M}$ presented no differences in serum samples from women with infected symptomatic fetuses, infected asymptomatic fetuses, and uninfected fetuses (Figure 2(d)).

\section{3. sHLA-G Concentration Gradient between Maternal Serum} and Amniotic Fluid. The sHLA-G increase in amniotic fluids of infected symptomatic fetuses prompted the question of whether sHLA-G was produced locally in amniotic compartment or derived from maternal blood. Fetal and maternal compartments are mutually interconnected and several molecules are exchanged through the amniotic and chorionic membrane. This molecular interchange could be hypothesized also for HLA molecules. Therefore we evaluated the concentration gradient between serum samples from primary infected women and the corresponding amniotic fluids.

A sHLA-G concentration gradient from the amniotic fluid to the maternal serum was observed only in infected symptomatic fetuses, while uninfected and infected asymptomatic fetuses presented an inverse sHLA-G gradient (Figure $3(\mathrm{a})$ ). These results suggest a local fetal production of sHLA-G, increased only in fetuses with symptomatic HCMV infection.

3.4. sHLA-G Index. The association between fetal HCMV infection and increased sHLA-G expression in amniotic fluid was confirmed calculating the sHLA-G index in comparison with albumin. Albumin is the most prevalent serum protein which surrounds the embryo and is detected in amniotic fluids. Fainardi et al. [27] reported the use of cerebrospinal fluid and serum albumin content to evaluate sHLA-G brain production. Since both blood-brain interface and placenta are considered selective barriers, we applied the same concept to quantify the fetal compartment production of sHLA-G, 


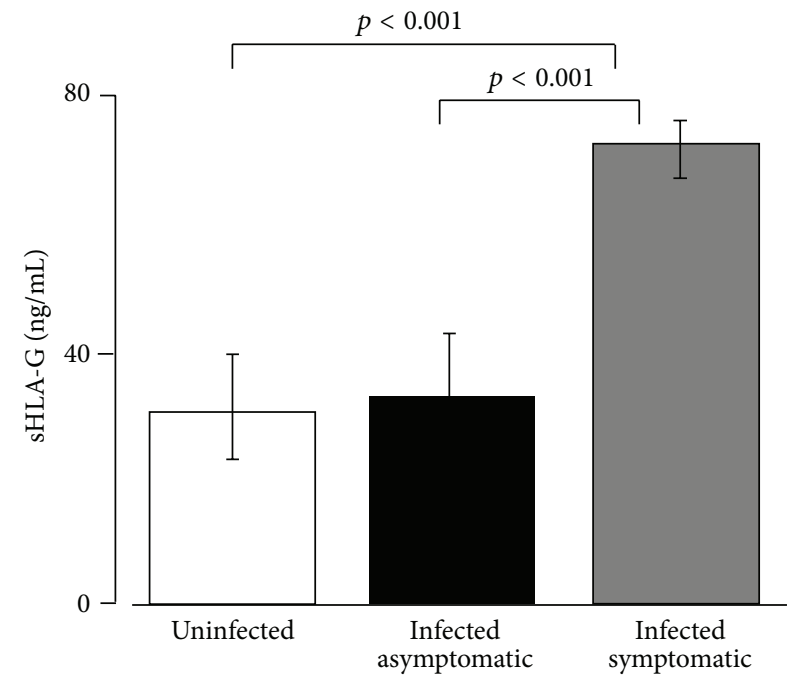

(a)

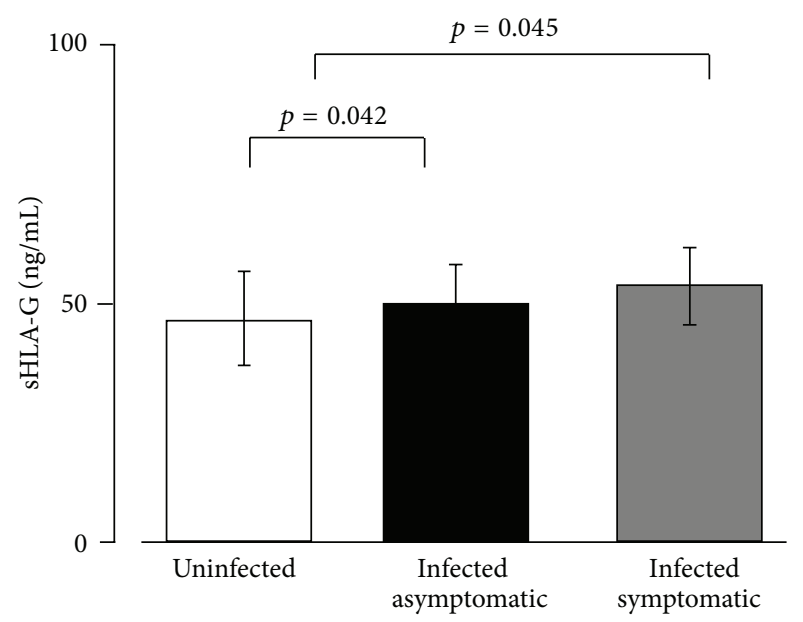

(c)

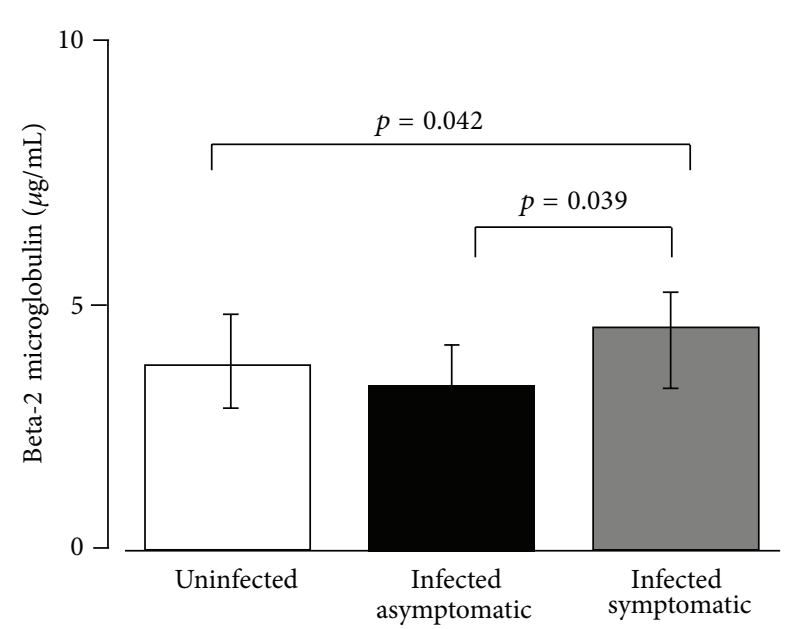

(b)

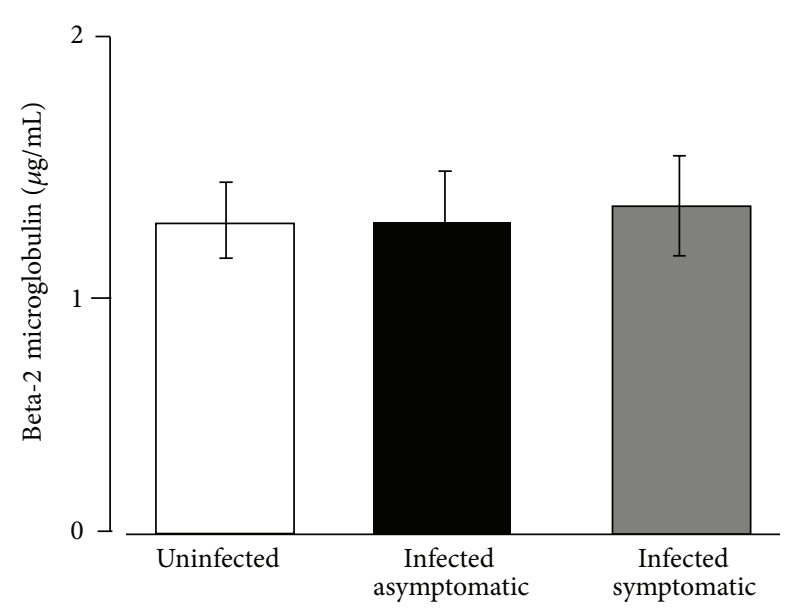

(d)

FIGURE 2: Amniotic fluid samples expression of (a) sHLA-G and (b) b2M molecules according to the fetal/neonatal outcome. Maternal serum samples expression of (c) sHLA-G and (d) b2M molecules according to the fetal/neonatal outcome. $p$ values obtained by Student's $t$-test and mean \pm standard deviation are reported.

evaluating the relative amount of amniotic sHLA-G and albumin compared with maternal serum levels. Any increase in the index could be ascribed to sHLA-G production in the fetal compartment. The highest sHLA-G indexes were detected in infected symptomatic fetuses (19.5\%) compared to infected asymptomatic fetuses (6\%) and uninfected (5.1\%) $(p<0.001, p<0.001$, resp.) (Figure 3(b)).

3.5. HLA-G Free-Heavy Chain Analysis. HLA-G can be expressed as b2M associated or free-heavy chain. Previous studies documented a different distribution of these two conformations at the maternal-fetus interface [29]. We evaluated the presence of HLA-G free-heavy chain in both sera and in amniotic fluids from primary HCMV infected pregnant women with asymptomatic or symptomatic fetus. HLA-G free-heavy chain was detected with a tendency to be more frequent in amniotic fluids from symptomatic fetuses $(p=$ $0.074)$. On the contrary, maternal sera did not present HLA$\mathrm{G}$ free-heavy chain (Table 1).

Representative examples of maternal serum and AF reactivity to antigens on the Western blot are shown in Figure 3(c). In lines 1,2 , and 3, we reported HLA-G positive samples analyzed for b2M associated form (MEM-G9 detection), while the same samples were analyzed for HLA-G free-heavy chain (MEM-G1 detection) in lines 4, 5, and 6.

3.6. sHLA-G Predictive Efficacy. We analyzed serum and amniotic fluid samples for sHLA-G and selected different cutoffs to be used as differentiation values. ROC analysis showed serum values above $50 \mathrm{ng} / \mathrm{mL}$ and amniotic values above $30 \mathrm{ng} / \mathrm{mL}$ with the highest sensitivity and specificity and an Area Under an ROC Curve of 0.83 and 0.86, respectively 


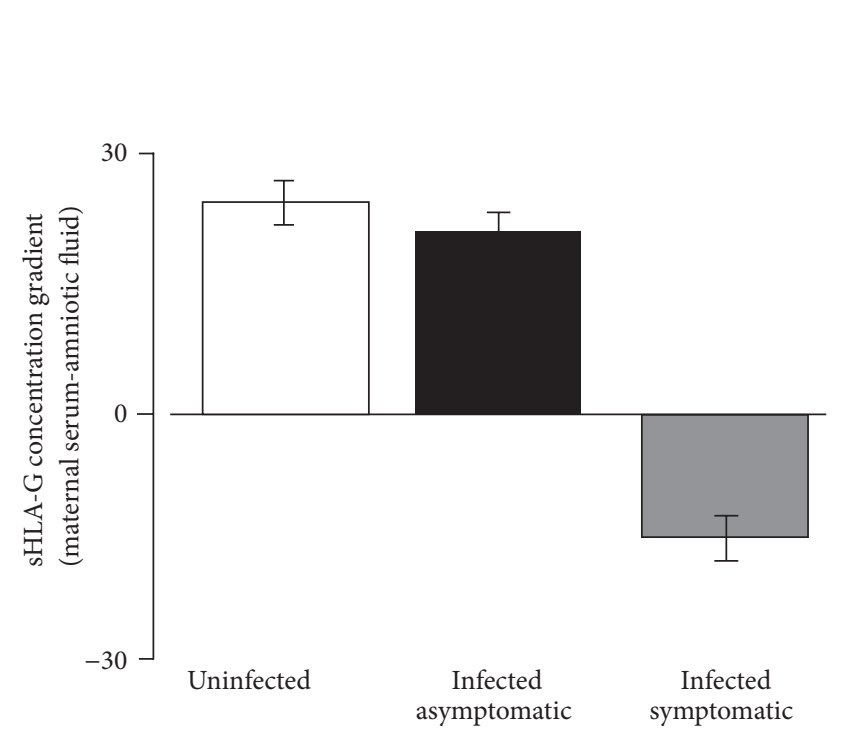

(a)

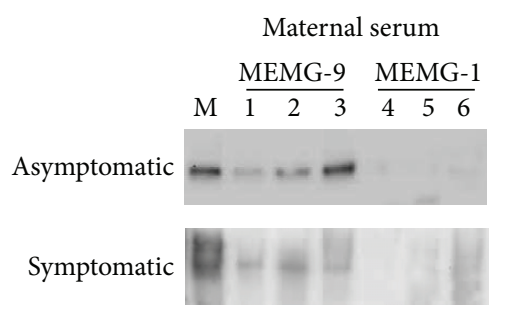

(c)

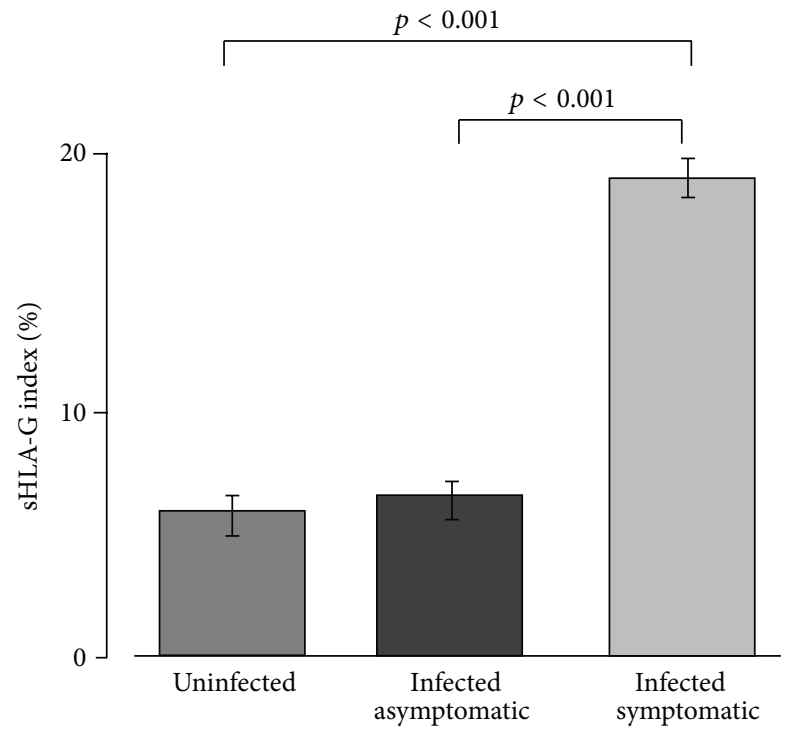

(b)

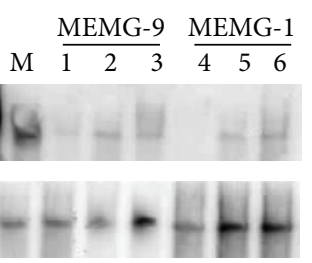

.

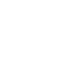

Figure 3: (a) sHLA-G concentration gradients according to the fetal/neonatal outcome. (b) sHLA-G indexes (\%) according to the fetal/neonatal outcome. (c) Representative Western blot analysis of maternal serum and amniotic fluids samples from HCMV primary infected pregnancy subdivided according to the fetal/neonatal outcome. The analysis were performed after immunoprecipitation with antib2M associated HLA-G moAb (MEM-G9, Exbio) (lines 1 to 3) and anti-free HLA-G HC moAb (MEMG-1, Exbio) (lines 4 to 6). JEG3 cell line supernatants were used as positive control (M) and the positivity for HLA-G molecule was evidenced at $39 \mathrm{kD}$.

TABle 1: Prognostic HLA-G biomarker of symptomatic congenital HCMV infection.

\begin{tabular}{|c|c|c|c|c|c|c|c|c|c|}
\hline \multirow{2}{*}{ Parameters } & \multirow{2}{*}{\multicolumn{2}{|c|}{ Cutoff }} & \multicolumn{2}{|c|}{ Fetuses } & \multicolumn{4}{|c|}{ Diagnostic accuracy } & \multirow{2}{*}{ AUA } \\
\hline & & & Sympt & Asympt & Sens\% & Spec\% & PPV\% & NPV\% & \\
\hline \multicolumn{10}{|l|}{ Maternal serum samples } \\
\hline \multirow{2}{*}{ HLA-G free-heavy chain } & & Presence & 0 & 0 & 0 & 100 & 0 & 29.4 & \multirow{2}{*}{0.5} \\
\hline & \multirow{3}{*}{$50 \mathrm{ng} / \mathrm{mL}$} & Absence & 12 & 5 & $0-26.7$ & $47.9-100$ & & $10.4-55.9$ & \\
\hline \multirow{2}{*}{ sHLA-G } & & Above & 10 & 0 & 83.3 & 100 & 100 & 71.4 & \multirow{2}{*}{0.83} \\
\hline & & Below & 2 & 5 & $51.6-97.4$ & $47.9-100$ & $68.9-100$ & $29.3-95.5$ & \\
\hline \multicolumn{10}{|l|}{ Amniotic fluid samples } \\
\hline \multirow{2}{*}{ HLA-G free-heavy chain } & & Presence & 12 & 3 & 100 & 40 & 80 & 100 & \multirow{2}{*}{0.79} \\
\hline & & Absence & 0 & 2 & $73.3-100$ & $6.4-84.6$ & $51.9-95.4$ & $19.2-100$ & \\
\hline \multirow{2}{*}{ sHLA-G } & \multirow{2}{*}{$30 \mathrm{ng} / \mathrm{mL}$} & Above & 11 & 0 & 91.7 & 100 & 100 & 83.3 & \multirow{2}{*}{0.86} \\
\hline & & Below & 1 & 5 & $61.5-98.6$ & $47.9-100$ & $71.3-100$ & $36.1-97.2$ & \\
\hline
\end{tabular}

Sens: sensitivity; Spec: specificity; PPV: positive predictive value; NPV: negative predictive value; AUA: Area Under an ROC Curve; Sympt: symptomatic; Asympt: asymptomatic. 
(Table 1), in differentiating symptomatic from asymptomatic congenital infections. Similarly, the presence of HLA-G freeheavy chain in amniotic fluids shows a high sensitivity and specificity and an Area Under an ROC Curve of 0.79 , in identifying symptomatic congenital infections. Logistic regression analysis excluded the presence of confounding variables.

\section{Discussion}

The prenatal diagnosis provides the optimal means for diagnosing HCMV fetal infection. The specificity is very good and the sensitivity depends on the kind of samples used (amniotic fluid $>$ fetal blood), the technique used (Real-Time PCRPolymerase Chain Reaction > viral culture), and the timing of the procedure with respect to the onset of maternal infection and the gestational age.

All literature data report that the amniotic fluid is the most appropriate material for the diagnosis of fetal HCMV infection. Positive results in amniotic fluid identify all HCMV infected fetuses (positive predictive value $=100 \%$ ) but do not identify the infants who will have symptoms at birth [6].

Although the highest median values of HCMV-DNA in amniotic fluid tend to indicate an increased risk of severe infection, high viral loads may be associated with symptomatic or asymptomatic congenital infections. Indeed, a correlation between the high HCMV load in amniotic fluid and fetal/neonatal outcome has not been demonstrated [6].

More recently, some studies have evoked the prognostic value of fetal viremia/viral load and/or level of specific IgM; however this remains controversial. It has been proposed that platelet count gives a better indication. New data has demonstrated that the determination of multiple markers (haematological, biochemical, and virological markers) in fetal blood following virus detection in amniotic fluid is predictive of perinatal outcome in fetuses with HCMV infection [21]. Further studies in a larger number of symptomatic cases should be performed to verify the prognostic efficacy of determination of multiple parameters in fetal blood.

In this work, we analyzed the levels of sHLA-G and b2M molecules in the maternal serum samples and showed that sHLA-G median levels were significantly higher in maternal serum from pregnant women with primary HCMV infection than in nonprimary and uninfected, respectively $(p<0.001$ and $p<0.006$ ). On the contrary, beta- 2 microglobulin levels were only slightly higher in maternal serum from pregnant women with primary HCMV infection than in nonprimary and uninfected, respectively ( $p=0.12$ and $p=0.06$ ). The differences in immune competence towards HCMV in primary infected and in nonprimary infected mothers could explain the different production of sHLA-G that can act as viral immune escape mechanism or as a tentative reduction of immune activation in primary HCMV infection, which is not induced in nonprimary HCMV infected women.

Furthermore, we evaluated whether sHLA-G molecules could be considered prognostic biomarkers of symptomatic congenital HCMV infection.

We observed that sHLA-G levels in both maternal serum and amniotic fluid samples are significantly related to symptomatic HCMV fetal infections as supported by ROC analysis (Table 1). In fact, sHLA-G levels above $50 \mathrm{ng} / \mathrm{mL}$ in maternal blood and $30 \mathrm{ng} / \mathrm{mL}$ in amniotic fluid are correlated with symptomatic HCMV congenital infections. High levels of sHLA-G in maternal and fetal compartments show a specificity of $100 \%$ for symptomatic congenital infection and a sensitivity ranging between 83 and $92 \%$.

The evidence that sHLA-G levels increase only in the presence of symptomatic fetuses suggests a specific fetal production of these molecules. We obtained confirmation of this hypothesis through (i) the evaluation of the concentration gradient, which is higher in the amniotic fluid versus the maternal blood in case of symptomatic infection; (ii) the sHLA-G indexes calculation, which support a fetal production; (iii) the HLA-G free-heavy chian, which is commonly expressed by distal trophoblasts [29], which is present only in amniotic fluid.

The increase of fetal HLA-G expression could be caused by HCMV-encoded proteins that are known to interact with HLA mRNAs and proteins, modifying their stability and secretory pathways [15-19]. The increase of HLA-G expression could enhance HCMV immune escape, increasing the risk of congenital infections and symptomatic sequelae. Our results suggest that serum and amniotic fluid sHLA-G might be an additional biomarker of congenital HCMV infection that could be considered in combination with currently used viral and biological markers [20,30], providing the best key to the reliable identification of fetuses at risk of congenital disease as well as of fetuses with a favorable outcome.

\section{Conclusions}

To the best of our knowledge, this is the first observation that considers the possible use of serum and amniotic fluid sHLA$\mathrm{G}$ as a biomarker to discriminate between symptomatic and asymptomatic HCMV congenital infection. However, future studies with larger cohort of fetuses should be performed in order to verify whether the addition of serum sHLA$\mathrm{G}$ determination to virologic markers may be crucial in identifying fetuses at highest risk of severe pathologies.

\section{Competing Interests}

All authors reported no competing interests.

\section{Acknowledgments}

The technical expertise of Francesca Bellini from the Laboratory of Virology, St. Orsola-Malpighi University Hospital, Bologna, is greatly acknowledged. The authors thank Iva Pivanti for technical assistance. They also thank their Linguistic Consultant, Lucy Scioscia, for editing the English language text. This work was partially supported by grants (SA43GABR) from St. Orsola-Malpighi University Hospital Bologna (Italy), Ricerca Finalizzata 2006 from Health Care Ministry, Rome (Italy), Ricerca Finalizzata 2010 from Health Care Ministry, Rome (Italy), Young Scientists award "A. Liberati” 2012 and 2013, Regione Emilia Romagna (Italy). 


\section{References}

[1] C. S. Peckham, "Cytomegalovirus infection: congenital and neonatal disease," Scandinavian Journal of Infectious Diseases, vol. 78, pp. 82-87, 1991.

[2] A. Kenneson and M. J. Cannon, "Review and meta-analysis of the epidemiology of congenital cytomegalovirus (CMV) infection," Reviews in Medical Virology, vol. 17, no. 4, pp. 253276, 2007.

[3] K. B. Fowler, S. Stagno, R. F. Pass, W. J. Britt, T. J. Boll, and C. A. Alford, "The outcome of congenital cytomegalovirus infection in relation to maternal antibody status," The New England Journal of Medicine, vol. 326, no. 10, pp. 663-667, 1992.

[4] J. J. C. de Vries, E. W. van Zwet, F. W. Dekker, A. C. M. Kroes, P. H. Verkerk, and A. C. T. M. Vossen, "The apparent paradox of maternal seropositivity as a risk factor for congenital cytomegalovirus infection: a population-based prediction model," Reviews in Medical Virology, vol. 23, no. 4, pp. 241-249, 2013.

[5] S. B. Boppana, R. F. Pass, W. J. Britt, S. Stagno, and C. A. Alford, "Symptomatic congenital cytomegalovirus infection: neonatal morbidity and mortality," The Pediatric Infectious Disease Journal, vol. 11, no. 2, pp. 93-99, 1992.

[6] T. Lazzarotto, B. Guerra, L. Gabrielli, M. Lanari, and M. P. Landini, "Update on the prevention, diagnosis and management of cytomegalovirus infection during pregnancy," Clinical Microbiology and Infection, vol. 17, no. 9, pp. 1285-1293, 2011.

[7] B. Guerra, G. Simonazzi, C. Puccetti et al., "Ultrasound prediction of symptomatic congenital cytomegalovirus infection," American Journal of Obstetrics and Gynecology, vol. 198, no. 4, pp. 380-e7, 2008.

[8] T. Lazzarotto, B. Guerra, M. Lanari, L. Gabrielli, and M. P. Landini, "New advances in the diagnosis of congenital cytomegalovirus infection," Journal of Clinical Virology, vol. 41, no. 3, pp. 192-197, 2008.

[9] S. Gouarin, E. Gault, A. Vabret et al., "Real-time PCR quantification of human cytomegalovirus DNA in amniotic fluid samples from mothers with primary infection," Journal of Clinical Microbiology, vol. 40, no. 5, pp. 1767-1772, 2002.

[10] T. Goegebuer, B. Van Meensel, K. Beuselinck et al., "Clinical predictive value of real-time PCR quantification of human cytomegalovirus DNA in amniotic fluid samples," Journal of Clinical Microbiology, vol. 47, no. 3, pp. 660-665, 2009.

[11] A. Lin, H. Xu, and W. Yan, "Modulation of HLA expression in human cytomegalovirus immune evasion," Cellular \& Molecular Immunology, vol. 4, no. 2, pp. 91-98, 2007.

[12] R. Rizzo, M. Vercammen, H. van de Velde, P. A. Horn, and V. Rebmann, "The importance of HLA-G expression in embryos, trophoblast cells, and embryonic stem cells," Cellular and Molecular Life Sciences, vol. 68, no. 3, pp. 341-352, 2011.

[13] R. Rizzo, A. Trentini, D. Bortolotti et al., "Matrix metalloproteinase-2 (MMP-2) generates soluble HLA-G1 by cell surface proteolytic shedding," Molecular and Cellular Biochemistry, vol. 381, no. 1-2, pp. 243-255, 2013.

[14] R. Rizzo, A. S. Andersen, M. R. Lassen et al., "Soluble human leukocyte antigen-G isoforms in maternal plasma in early and late pregnancy," American Journal of Reproductive Immunology, vol. 62 , no. 5 , pp. 320-338, 2009.

[15] Y. Jun, E. Kim, M. Jin et al., "Human cytomegalovirus gene products US3 and US6 down-regulate trophoblast class I MHC molecules," Journal of Immunology, vol. 164, no. 2, pp. 805-811, 2000.

[16] M. Onno, C. Pangault, G. Le Friec, V. Guilloux, P. André, and R. Fauchetz, "Modulation of HLA-G antigens expression by human cytomegalovirus: specific induction in activated macrophages harboring human cytomegalovirus infection," The Journal of Immunology, vol. 164, no. 12, pp. 6426-6434, 2000.

[17] B. Park, E. Spooner, B. L. Houser, J. L. Strominger, and H. L. Ploegh, "The HCMV membrane glycoprotein US10 selectively targets HLA-G for degradation," The Journal of Experimental Medicine, vol. 207, no. 9, pp. 2033-2041, 2010.

[18] M. T. Barel, M. Ressing, N. Pizzato et al., "Human cytomegalovirus-encoded US2 differentially affects surface expression of MHC class I locus products and targets membrane-bound, but not soluble HLA-G1 for degradation," The Journal of Immunology, vol. 171, no. 12, pp. 6757-6765, 2003.

[19] D. J. Schust, D. Tortorella, J. Seebach, C. Phan, and H. L. Ploegh, "Trophoblast class I major histocompatibility complex (MHC) products are resistant to rapid degradation imposed by the human cytomegalovirus (HCMV) gene products US2 and US11," The Journal of Experimental Medicine, vol. 188, no. 3, pp. 497-503, 1998.

[20] E. Fabbri, M. G. Revello, M. Furione et al., "Prognostic markers of symptomatic congenital human cytomegalovirus infection in fetal blood," BJOG: An International Journal of Obstetrics and Gynaecology, vol. 118, no. 4, pp. 448-456, 2011.

[21] L. Gabrielli, M. P. Bonasoni, D. Santini et al., "Congenital cytomegalovirus infection: patterns of fetal brain damage," Clinical Microbiology and Infection, vol. 18, no. 10, pp. E419E427, 2012.

[22] M. Lanari, T. Lazzarotto, V. Venturi et al., "Neonatal cytomegalovirus blood load and risk of sequelae in symptomatic and asymptomatic congenitally infected newborns," Pediatrics, vol. 117, no. 1, pp. e76-e83, 2006.

[23] T. Lazzarotto, A. Ripalti, G. Bergamini et al., "Development of a new cytomegalovirus (CMV) immunoglobulin M (IgM) immunoblot for detection of CMV-specific IgM," Journal of Clinical Microbiology, vol. 36, no. 11, pp. 3337-3341, 1998.

[24] T. Lazzarotto, L. Gabrielli, M. P. Foschini et al., "Congenital cytomegalovirus infection in twin pregnancies: viral load in the amniotic fluid and pregnancy outcome," Pediatrics, vol. 112, no. 2, pp. e153-e157, 2003.

[25] R. Rizzo, B. Fuzzi, M. Stignani et al., "Soluble HLA-G molecules in follicular fluid: a tool for oocyte selection in IVF?" Journal of Reproductive Immunology, vol. 74, no. 1-2, pp. 133-142, 2007.

[26] E. Fainardi, R. Rizzo, L. Melchiorri et al., "Soluble HLA-G molecules are released as HLA-G5 and not as soluble HLA-G1 isoforms in CSF of patients with relapsing-remitting multiple sclerosis," Journal of Neuroimmunology, vol. 192, no. 1-2, pp. 219225, 2007.

[27] E. Fainardi, R. Rizzo, L. Melchiorri et al., "Intrathecal synthesis of soluble HLA-G and HLA-I molecules are reciprocally associated to clinical and MRI activity in patients with multiple sclerosis," Multiple Sclerosis, vol. 12, no. 1, pp. 2-12, 2006.

[28] E. Fainardi, R. Rizzo, L. Melchiorri et al., "Presence of detectable levels of soluble HLA-G molecules in CSF of relapsingremitting multiple sclerosis: relationship with CSF soluble HLA-I and IL-10 concentrations and MRI findings," Journal of Neuroimmunology, vol. 142, no. 1-2, pp. 149-158, 2003. 
[29] P. J. Morales, J. L. Pace, J. S. Platt, D. K. Langat, and J. S. Hunt, "Synthesis of $\beta(2)$-microglobulin-free, disulphide-linked HLAG5 homodimers in human placental villous cytotrophoblast cells," Immunology, vol. 122, no. 2, pp. 179-188, 2007.

[30] M. Leruez-Ville, J. Stirnemann, Y. Sellier et al., "Feasibility of predicting the outcome of fetal infection with cytomegalovirus at the time of prenatal diagnosis," American Journal of Obstetrics and Gynecology, 2016. 


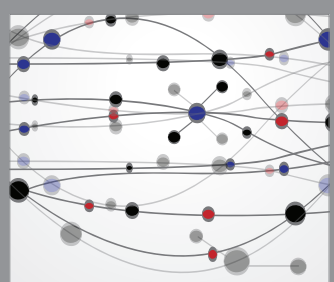

The Scientific World Journal
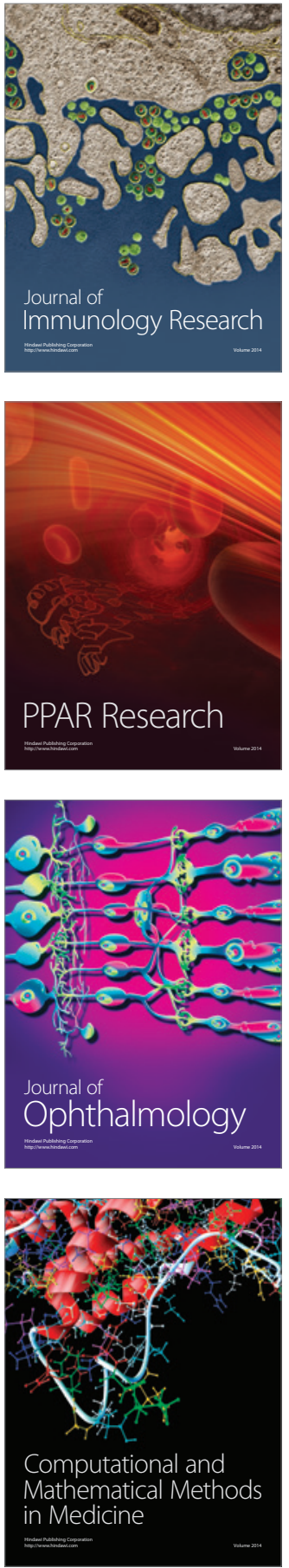

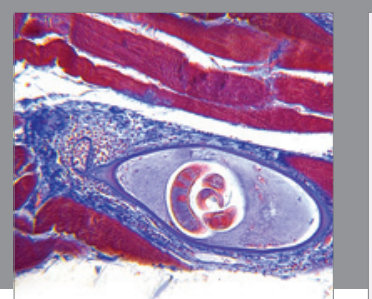

Gastroenterology Research and Practice

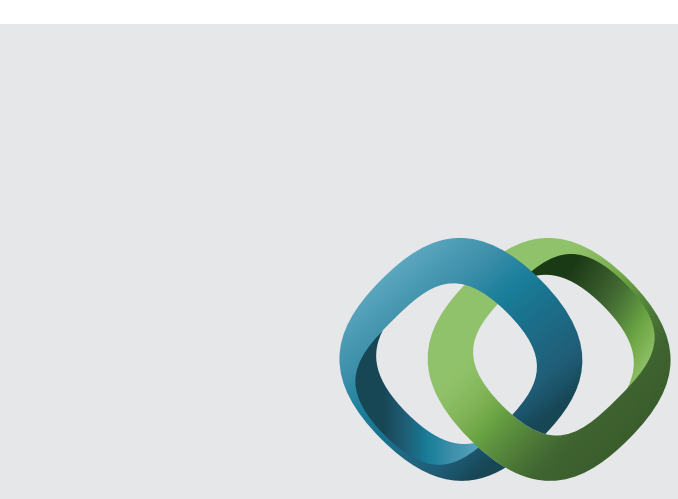

\section{Hindawi}

Submit your manuscripts at

http://www.hindawi.com
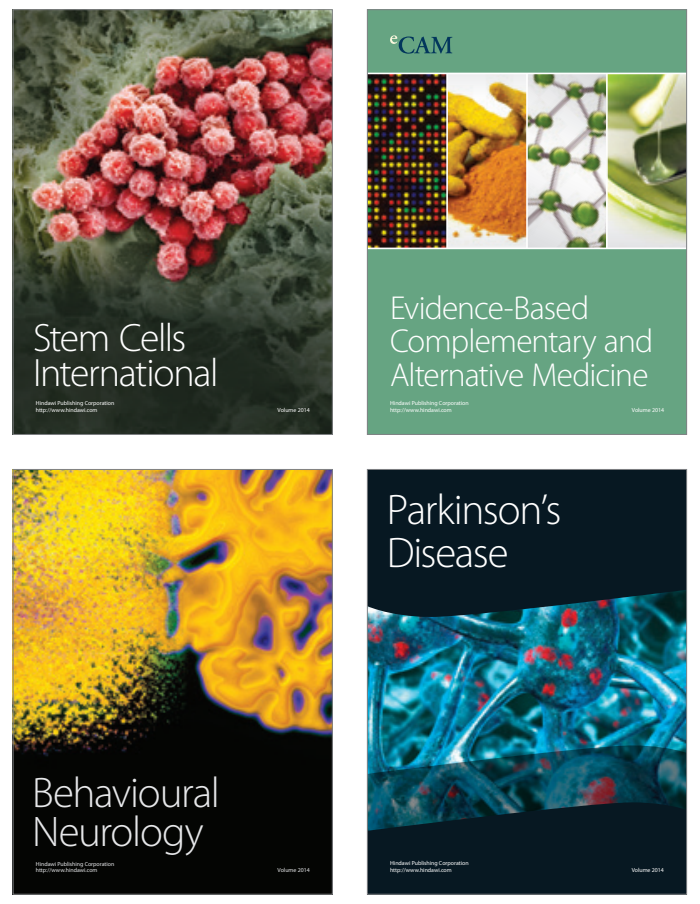
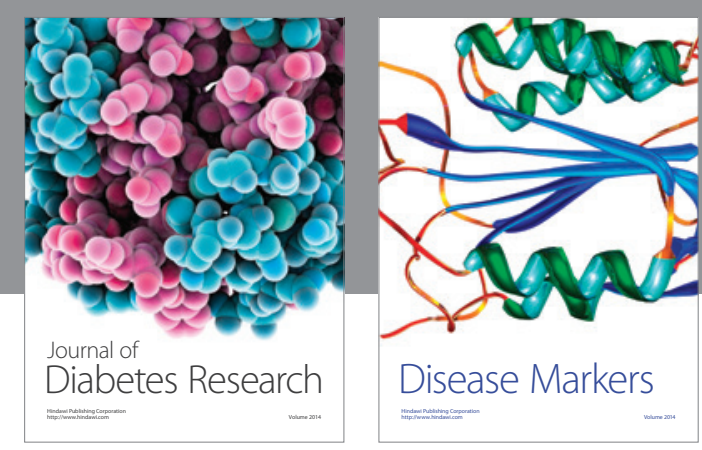

Disease Markers
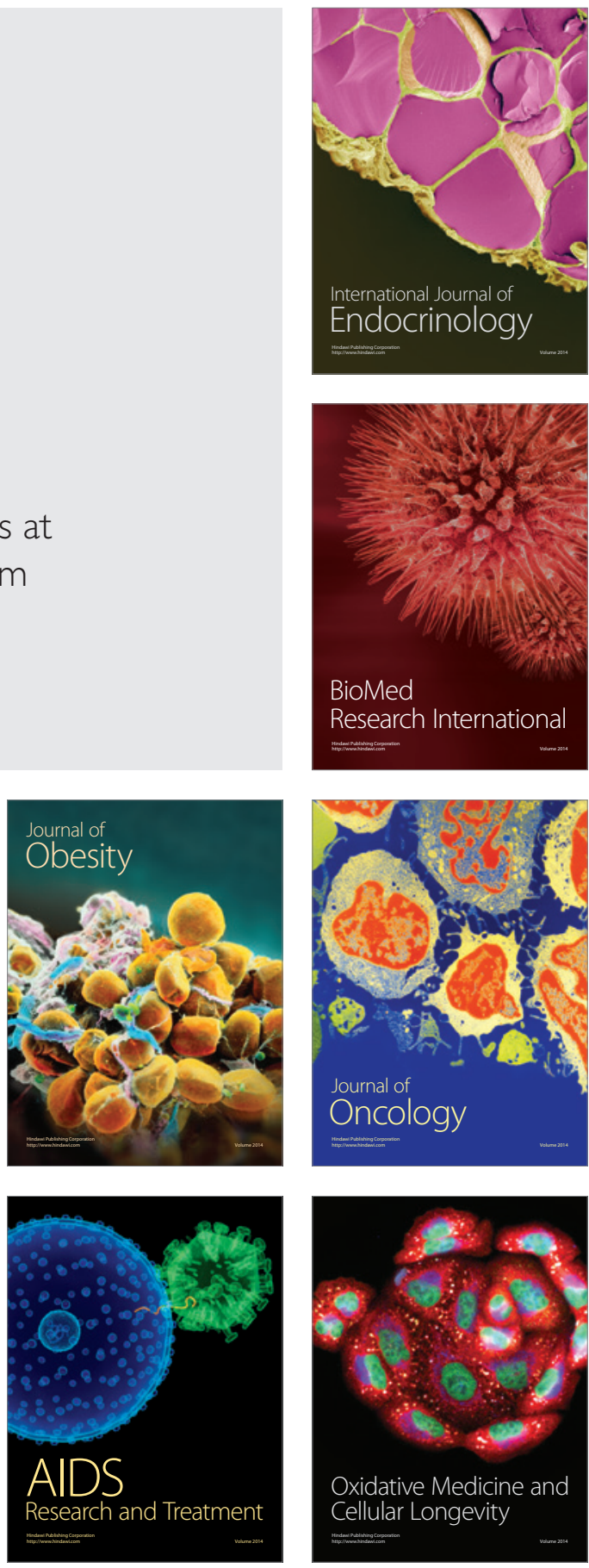